Cahiers $d u$ MONDE RUSSE

\section{Cahiers du monde russe}

Russie - Empire russe - Union soviétique et États indépendants

$56 / 4 \mid 2015$

Médiateurs d'empire en Asie centrale (1820-1928)

\title{
Alexandre Sumpf, La Grande Guerre oubliée, Russie 1914-1918
}

\section{Cloé Drieu}

\section{OpenEdition}

\section{Journals}

Édition électronique

URL : http://journals.openedition.org/monderusse/8252

DOI : $10.4000 /$ monderusse. 8252

ISSN : $1777-5388$

\section{Éditeur}

Éditions de l'EHESS

\section{Édition imprimée}

Date de publication : 1 octobre 2015

Pagination : 832-837

ISBN : $978-2-7132-2507-9$

ISSN : 1252-6576

Référence électronique

Cloé Drieu, «Alexandre Sumpf, La Grande Guerre oubliée, Russie 1914-1918 », Cahiers du monde russe [En ligne], 56/4 | 2015, mis en ligne le 01 octobre 2015, Consulté le 23 septembre 2020. URL : http:// journals.openedition.org/monderusse/8252; DOI : https://doi.org/10.4000/monderusse.8252

Ce document a été généré automatiquement le 23 septembre 2020 


\title{
Alexandre Sumpf, La Grande Guerre oubliée, Russie 1914-1918
}

\author{
Cloé Drieu
}

\section{RÉFÉRENCE}

Alexandre SUMPF, La Grande Guerre oubliée, Russie 1914-1918, Paris : Perrin, 2014, $527 \mathrm{p}$.

1 Avec cet ouvrage, Alexandre Sumpf réintégre la Russie dans une historiographie de la Grande Guerre (centrée essentiellement sur l'Europe et les fronts dits " primaires »). Pour faciliter la comparaison, l'auteur s'est borné au cadre chronologique européen, celui des années 1914-1918. Ce cadre lui permet également d'inclure dans son propos le début de la guerre civile et de respecter ainsi les logiques spécifiquement russes du conflit. Après une courte introduction, qui aurait mérité de situer davantage son ouvrage dans l'historiographie actuelle de l'Empire russe pendant le conflit mondial et de celle de la transition vers la guerre civile - on pense, sans exhaustivité, aux travaux d'Eric Lohr, de Joshua Sanborn, de Peter Holquist ou de Peter Gatrell, lesquels sont bien évidemment cités dans le corps du texte -, La Grande Guerre oubliée s'ouvre sur un premier chapitre intitulé «La Russie s'en va-t'en guerre » (p.16-67), à la croisée de l'histoire diplomatique,

militaire et sociopolitique. Ce chapitre, basé sur des sources secondaires et des sources primaires éditées, définit le contexte historique général, et évoque les responsabilités de la Russie dans le déclenchement de la guerre, l'union nationale autour des Romanov, la capacité du pays à tenir dans un conflit de longue durée. L'auteur reprend les débats sur les causes et les processus de l'entrée en guerre du pays (F. Fischer, McMeekin, C. Clark) et en situe les enjeux généraux : enjeux historiques comme l'impact de la défaite dans la guerre russo-japonaise et enjeux diplomatiques comme les jeux d'alliances militaires (notamment pour le contrôle des détroits). Le chapitre se termine par un portrait synthétique de la situation politique et économique interne de l'Empire russe (rôles du panslavisme et des élites commerciales et militaires d'origine 
allemande, d'un patriotisme réel, mais peu ancré) et de sa situation militaire (soldats et officiers, faiblesse de l'armement, description des fronts et des grandes phases de la guerre, organisation de la mobilisation et résistances à diverses périodes).

Puis, l'auteur s'interroge sur l'expérience de guerre des soldats et les efforts économiques suscités par cette guerre. Basé sur des témoignages, des carnets de guerre de soldats et d'officiers publiés et des données d'archives (conservées au RGVIA) - qui restent proportionnellement moins nombreux que ceux de leurs camarades français du fait d'un analphabétisme plus important et d'un système postal moins développé -, le deuxième chapitre "Le théâtre de la guerre " (p.69-112) offre une contribution originale et vivante sur le quotidien des soldats en guerre, partagé par plus de 16 millions d'hommes mobilisés. Confrontés à l'expérience du feu et à la routine du front, au déracinement, au dépaysement et à une adaptation constante à de nouveaux repères spatiaux, temporels, moraux et politiques (p.74), les soldats - l'auteur se focalise essentiellement sur les soldats d'origine russe - semblent davantage livrés à eux-mêmes que leurs homologues européens. Mal nourris (la ration décline constamment tout au long du conflit), mal équipés (un tiers des effectifs de l'armée se voit attribuer des bottes fourrées pour l'hiver 1915, p. 82- 83), mal commandés (du fait, notamment, de la mauvaise coordination entre l'état-major et les officiers sur le terrain), ils sont plus enclins à la fraternisation ou à la désertion, ce qui constitue l'une des préoccupations principales de l'état-major impérial. Quant aux populations civiles, elles sont soumises à l'omnipotence des militaires, notamment en territoires conquis, à la loi martiale, aux réquisitions, à la suspicion (c'est le cas des populations d'origines juive ou allemande qui sont déplacées dès le début de la guerre) et aux violences des soldats, en particulier des cosaques. Dans le chapitre 3, "Les coulisses de la guerre " (p.113-167), l'auteur détaille l'effort de guerre dans son ensemble, et d'abord sur le plan économique. L'adaptation de la production agricole et industrielle (armement, munitions) nécessite des efforts coûteux qui mobilisent les secteurs public et privé et sont lourds de conséquences pour la société. La situation de la production est aggravée par la Grande Retraite et la perte des territoires occidentaux de l'Empire russe (Pologne et pays Baltes) dont l'activité industrielle était très dynamique (fer, acier et chimie) ; le transfert partiel des industries et l'évacuation des réfugiés ne suffisent pas à combler les pertes de ces territoires. De plus, le pays est plongé dans un état de disette constant, dû à la réquisition des animaux, aux livraisons obligatoires, mais aussi à la gabegie de l'armée. C'est également un moment où le gouvernement a largement recours au travail contraint ce qui, cumulé avec les conditions de travail et de vie déplorables, comme le montrent certains passages riches en couleur sur la pénurie et la prohibition d'alcool (p.161-164), engendre de vives tensions. Les femmes sont sollicitées et acquièrent pendant la guerre un véritable statut social tant en milieu rural qu'urbain leur proportion dans l'industrie passe de $30 \%$ à $40 \%$ (p.133) -, tout comme les prisonniers de guerre, employés principalement dans l'industrie (1,64 million au total), les champs et les chemins de fer. Même si ces derniers bénéficient d'un traitement relativement correct de la part de l'administration et de la société, ils connaissent un taux de mortalité au moins 2,5 fois plus élevé que dans les pays d'Europe (p. 137).

3 La guerre impose aux hommes et aux femmes une violence sans commune mesure avec celle qu'ils avaient vécue par le passé ; c'est cet état de guerre dans la société - à la fois violence et mobilisation - qui fait l'objet du troisième moment du livre. Le chapitre 4, "Les épreuves de la guerre " (p.169-211) présente les différentes facettes des 
violences de guerre. La mort de masse, d'abord, et les deuils difficiles, car, comme l'auteur le souligne, la Russie est le pays qui compte le plus de disparus (un quart du 1,89 million de pertes définitives au combat), par rapport aux autres puissances européennes, phénomène amplifié par le fait que les soldats ne disposent que rarement d'une plaque d'identification ou d'un livret militaire. Les grands déplacements de populations ensuite, ce qui comprend, outre le déplacement des hommes mobilisés, les déportations de populations suspectes (juives, allemandes, tsiganes), le mouvement des prisonniers de guerre tant russes qu'ennemis, ainsi que celui des réfugiés qui viennent grossir la population des villes. L'occupation du territoire, enfin, par des puissances étrangères (expérience de l'Ober Ost). Face à l'ampleur de la situation et au chaos généralisé, les moyens dont disposent l'État et les intellectuels russes pour mobiliser les populations (chapitre 5 : «Une société mobilisée ») se développent rapidement et intensément, qu'il s'agisse de la presse, de l'ensemble des supports d'information et de propagande (brochures, lubki et périodiques militaires) ou encore de la littérature de guerre et de la propagande cinématographique, à l'arrière comme au front. S'ajoutent à tout ceci les initiatives nationales locales et communautaires d'une extrême diversité, dans le domaine de la bienfaisance et de l'assistance (p.248); la force de l'« auto-mobilisation sociale » est pour l'auteur une caractéristique russe qui peut être perçue comme un remède à la défaillance du système étatique impérial, lequel ne résistera pas à l'épreuve de la Grande Guerre.

Ainsi, dans un dernier moment du livre, l'auteur revient sur la période révolutionnaire, l'effondrement de l'Empire russe, et aussi sur l'absence de mémoire qu'a suscitée la Grande Guerre dans l'aire soviétique (chapitre 6: "La dissolution de la nation impériale »). Les efforts de mobilisation patriotique (aides aux réfugiés, aux blessés, aux familles), portés par des communautés locales (zemstva) ou nationales donnent naissance à une nouvelle élite technocratique, perçue par l'État comme une menace. L'émergence d'une société civile et politique se manifeste également par les élans révolutionnaires de février 1917 (5 au 12 mars), qui donnent naissance à une opposition politique multiple (Douma, partis radicaux, et soviets), par la réaction sévère du tsar et, de fait, par un antimonarchisme croissant et de plus en plus général. L'éclosion des consciences nationales au sein des territoires occidentaux de l'Empire russe (Biélorussie, Pologne, Ukraine, pays Baltes, Finlande), qui sont majoritairement des zones de front, doit beaucoup aux déplacements massifs de réfugiés et à leur catégorisation, voire leur stigmatisation nationale, ce qui en retour cristallise l'identité russe. L'année 1917 donne donc naissance à une série de bouleversements politiques et sociaux cristallisés par la révolution de Février (chute de la monarchie et nomination $\mathrm{du}$ gouvernement provisoire) puis par la révolution d'Octobre et la guerre civile (chapitre 7, "Le "catalyseur" de la Révolution ", p. 317-370 et chapitre 8, " De la guerre impérialiste à la guerre civile ", p. 371-421). Cette tourmente révolutionnaire et la politisation des esprits et des pratiques se lisent à tous les niveaux de la société russe en guerre (les villes de province, le monde agricole en proie aux troubles et aux violences) et, bien sûr, au sein de l'armée, comme le décrit l'auteur. Soldats comme officiers sont marqués en profondeur par la chute de l'autorité monarchique et la formation de comités qui s'ensuit, ce qui accélère le délitement de l'autorité et des hiérarchies militaires traditionnelles. Dans un ultime chapitre ("Une guerre oubliée ", p. 423-469), A. Sumpf s'intéresse aux «mémoires» de l'événement (mémoires officielles et privées, historiographies), ou en l'absence de celles-ci, à la représentation 
de la guerre dans les films (jusqu'à la Seconde Guerre mondiale), dans la production des historiens soviétiques ou encore dans la littérature.

5 L'ouvrage d'Alexandre Sumpf est un livre important car, basé en grande partie sur un travail en archives et nombre de sources primaires, il propose une vue générale de l'expérience de la Grande Guerre en Russie, qu'il est l'un des premiers à replacer dans l'historiographie occidentale du conflit, tout ou au moins dans l'historiographie de langue française. Toutefois, outre des imprécisions (utilisation aléatoire du calendrier julien et du calendrier grégorien, annonce d'un ouvrage en trois parties qui ne sont reprises ni dans la table des matières ni dans le corps de l'ouvrage), la synthèse proposée par l'auteur ne s'inscrit pas suffisamment dans les débats qui structurent les études de la Russie dans la tourmente des guerres et des révolutions, et ne se situe pas plus clairement par rapport à ces derniers. On pense à titre d'exemple aux travaux de Joshua Sanborn qui voit l'impact des mouvements de populations largement désocialisées (déplacements forcés, réfugiés, mobilité des militaires, etc.), comme une précondition de la guerre civile ${ }^{1}$; à ceci s'ajoute le poids d'une élite politique et militaire bolchevique ambitieuse qui, elle, sait « re-solidariser " grâce à l'institution militaire et à l'esprit de corps. Une interprétation plus poussée des violences de guerre - en s'inspirant des débats sur les violences et la culture de guerre qui ont mobilisé les chercheurs en France par exemple - aurait également permis de s'interroger sur l'impact de ces pratiques mises en œuvre en temps de guerre sur les politiques de l'État stalinien et des sociétés, qui ne sont véritablement jamais totalement "pacifiées " avant le milieu des années 1950. À côté de ces deux premières critiques, une autre est sans doute plus substantielle. Pourquoi ne pas considérer, à l'instar de J. Sanborn encore, - et de façon plus provocatrice -, les révoltes de l'été 1916 en Asie centrale, surtout dans les steppes, comme le début des guerres civiles dans l'espace impérial russe ? En fait, l'auteur se focalise sur les territoires occidentaux de l'Empire russe en guerre et, comme il le dit en introduction, sur "l'expérience de guerre des Russes " alors qu'il prétend faire l'« histoire totale " d'une " guerre totale » (p.15) ; il ne consacre de fait au Caucase et à l'Asie centrale que trois pages. Ainsi les minorités nationales ou ethniques caucasiennes et centrasiatiques, comme la catégorie des musulmans de l'Empire sont de fait les oubliées de la Grande Guerre oubliée - alors qu'elles représentent, du moins pour les populations musulmanes turciques conscrites dans l'Armée impériale (Tatars, Bashkir...), près de $10 \%$ du contingent militaire impérial ${ }^{2}$, et sont aussi victimes ou actrices des violences de guerre, des massacres, des mouvements de populations et de l'effort de mobilisation. Le front caucasien de la Grande Guerre n'est par exemple pas abordé ; l'ouvrage de Michael Reynolds ${ }^{3}$ aurait pu constituer à ce titre une source précieuse. Qu'est-ce qu'un empire en guerre si l'on en exclut ses minorités et ses propres fronts orientaux ? Vu l'ampleur et la complexité de ces questions, s'il n'était pas possible de les approfondir, au moins aurait-il été souhaitable de les esquisser, même succinctement.

Le parti pris de l'auteur a donc été celui d'une tentative de réintégration de l'Empire russe, mais amputé de ses espaces coloniaux, au sein des nations du "premier monde » dans la guerre en se focalisant sur le front russe, les combats et l'expérience de guerre des populations et des territoires " européens " parce que slaves. En fait, la question fondamentale que pose l'ouvrage d'Alexandre Sumpf est bien celle de savoir comment écrire l'histoire de la Grande Guerre dans des espaces considérés comme " périphériques ", et la Russie ne fait pas exception. "Périphériques " parce qu'« en retard ", puisque le champ historiographique relatif au premier conflit mondial ne 
s'est constitué que récemment à cause de la suprématie idéologique du mythe d'Octobre qui a lesté l'histoire russe et soviétique pendant des décennies ; " périphériques ", ensuite, parce qu'il est impossible, malgré quelques tentatives, de transposer vraiment les méthodes et les questionnements qui travaillent l'historiographie occidentale de la même manière sur les mondes russe et soviétique ; "périphériques ", enfin, parce que les historiens des " deuxième " et " troisième " mondes ont souvent pris comme jalon de référence l'expérience européenne de la guerre, sa chronologie avec ses déclarations de guerre et ses traités de paix, pour s'interroger sur les points communs ou les différences, tout en travaillant avec un handicap de taille puisque les sources testimoniales sont loin d'être aussi prolixes. Que faire ? S'il faut très certainement dire "Adieu à l'Europe " ${ }^{4}$ pour réinventer une histoire du premier conflit mondial et espérer créer un champ autonome de recherche, il reste encore à savoir comment le faire. Comment, d'une part, faire dialoguer les historiographies sans s'inscrire dans une réflexion binaire (points communs/ divergences) et comment, d'autre part, éviter de développer des champs de recherche complètement hermétiques les uns aux autres ? La piste de réflexion sur les zones de friction entre empires (shatterzones) ouverte par Omer Bartov et Eric D. Weitz ${ }^{5}$ ou même, encore, la démarche de Timothy Snyder ${ }^{6}$ sur la Seconde Guerre mondiale, malgré les critiques, peuvent être une direction à suivre et à développer ; une réflexion poussée sur les transformations de l'État et des systèmes de domination, le maintien ou l'émergence de (nouvelles) élites, la cooptation, le rôle ou l'évincement des notabilités à travers les tourmentes des révoltes, des révolutions et des guerres, civiles ou pas, en serait une deuxième; une troisième, peut-être moins originale mais pourtant fructueuse - suivie en partie dans l'ouvrage d'A. Sumpf -, aurait trait au nationalisme et à la (trans)formation des identités ethniques et nationales... La liste reste ouverte, très certainement pour longtemps encore, et les propositions bienvenues.

\section{NOTES}

1. En particulier Joshua Sanborn, «Unsettling the Empire : Violent Migrations and Social Disaster in Russia During World War I», The Journal of Modern History, 77, juin 2005, p. 290-324, repris dans son Imperial Apocalypse (2014).

2. Salavat Iskhakov, "Tjurki-musul'mane v rossiiskoi armii (1914-1917)», D. Vasil'ev, S. ljashtornyj, V. Trepavlov, dir., Tjurkologicheskij Sbornik, SPb., :Vostochnaja literatura RAN, 2003, p. 245-280.

3. M. Reynolds, Shattering Empires : The Clash and Collapse of the Ottoman and Russian Empires, 1908-1918, Cambridge-New York : Cambridge University Press, 2001.

4. Olivier Compagnon, L'Adieu à l'Europe, L'Amérique latine et la Grande Guerre (Argentine et Brésil, 1914-1939), P. : Fayard, 2013.

5. Omer Bartov, Eric D. Weitz, dir., Shatterzone of Empires: Coexistence and Violence in the German, Habsburg, Russian and Ottoman Borderlands, Bloomington-Indianapolis: Indiana University Press, 2013. 
6. Timothy Snyder, Terres de sang, L'Europe entre Hitler et Staline, traduit de l'anglais par Pierre-Emmanuel Dauzat, P. : Gallimard, 2012.

\section{AUTEURS}

CLOÉ DRIEU

CNRS-Cetobac 